\title{
Beneficios de la actividad física para mantener la capacidad funcional de los adultos mayores.
}

Benefits of physical activity to enhance the functional capacity of the elderly.

\section{Duque-Fernández, Leonor Mariana ${ }^{1}$, Ornelas Contreras, Martha ${ }^{1}$ y Benavides Pando, Elia Verónica ${ }^{1}$}

${ }^{1}$ Universidad Autónoma de Chihuahua. Paseos de Chihuahua, Chihuahua, México C.P. 31125.

\section{CORRESPONDENCIA}

Leonor Mariana Duque-Fernández:

leonormariana6@gmail.com

Universidad Autónoma de Chihuahua
CÓMO CITAR

Duque, L. M., Ornelas, M. \& Benavides, E. V. (2020). Beneficios de la actividad física para mantener la capacidad funcional de los adultos mayores. Revista de Ciencias del Ejercicio, 15 (1), pp. 1-19. Recuperado de: revistafod.uanl.mx

\section{RESUMEN}

Objetivo: Analizar por medio de una revisión sistemática la relación entre la actividad física y la capacidad funcional en adultos mayores.

Método: Se realizó una búsqueda electrónica en las siguientes bases de datos: Scopus, Scientific Electronic Library Online (Scielo), Web of Science (WOS) y PEDro, además se utilizó el motor de búsqueda Google Académico. En la búsqueda se emplearon las palabras clave functional capacity, physical activity, elderly, actividad física, envejecimiento, capacidad funcional, adulto mayor, programas de actividad física, en artículos publicados entre el 2008 y 2018, escritos en español o inglés.

Resultados: Se encontraron 5200 estudios, de los cuales se seleccionaron 53 estudios y todos ellos cumplieron con los criterios de inclusión. Con los artículos identificados, se procedió a revisar sus títulos, se eliminaron las dúplicas y se examinaron los ejes de estudio, con particular atención a la metodología propuesta, los resultados y la discusión. De los 53 artículos seleccionados se encuentra una gran prevalencia de estudios que reportan que el envejecimiento asociado a una buena ejecución de actividad física brinda beneficios en la capacidad funcional en el adulto mayor a través de diversos programas realizados. Conclusión: La actividad física en los adultos mayores debe ser considerada como una de las principales estrategias de mantenimiento de la capacidad funcional, promoción de la salud y prevención de la enfermedad. Es el único método probado y seguro que puede mantener la condición física de un individuo, además de impedir e incluso revertir los cambios en la composición corporal asociados al envejecimiento.

Palabras clave: Actividad física, capacidad funcional, adultos mayores.

\section{ABSTRACT}

Objective: To analyze by means of a systematic review the relationship between physical activity and functional capacity in older adults.

Method: An electronic search was carried out in the following databases: Scopus, Scientific Electronic Library Online (SciELO), Web of Science (WOS) and PEDro, in addition the Google Academic search engine was used. The following words were used in the search functional capacity, physical activity, elderly, physical activity, aging, functional capacity, older adult, physical activity programs, works published between 2008 and 2018, written in Spanish or English.

Results: 5200 studies were found, of which 53 studies were selected and they all met the inclusion criteria. With the identified articles, we proceeded to review the study titles, duplicates were eliminated or duplicates were removed and the axes of study were examined, with special attention to the proposed methodology, results and discussion. Of the 53 selected articles, there is a high prevalence of studies that report that aging associated with a good execution of physical activity provides benefits in functional capacity in the elderly through the various programs conducted. Conclusions: Physical activity in older adults it must be considered as one of the main strategies for maintaining functional capacity, promoting health and preventing disease. Physical activity is the only proven and safe method that can maintain the physical condition of an individual, in addition to preventing and even reversing changes in body composition associated with aging

Key words: Functional capacity, physical activity, elderly. 


\section{Introducción}

La actividad física realizada de forma periódica ha demostrado que contribuye a mejorar el estado de ánimo, el aislamiento social, el mal dormir y mejora el tratamiento de la osteoporosis (Araya, 2012).

Es inminente que los seres humanos pasen por el proceso de envejecimiento, éste va enlazado al estilo de vida que se haya llevado en los años previamente vividos. Estas características marcan el estrecho paso de la adultez a la adultez mayor: la disminución de la fuerza y de la flexibilidad, la reducción de la longitud del paso de la marcha y una desequilibrada función motriz. Hábitos sistemáticos a lo largo de la vida predeterminan la clase de adulto mayor que se quiere ser; la mala alimentación, la ingesta del alcohol, el consumo de tabaco y sobre todo, el sedentarismo son causas principales de un mal envejecimiento. Algunos otros como la actividad física regular, la buena alimentación y la estabilidad emocional, beneficiarán la salud física y mental del adulto mayor (Álvarez \& Alud, 2018).

Debido a las características anteriormente mencionadas es que existe la necesidad de envejecer activamente, concepto que es definido por la OMS (Organización Mundial de la Salud (OMS), 2015) como el proceso de optimización de oportunidades de salud, participación y seguridad con el objetivo de mejorar la calidad de vida en esta etapa.

La funcionalidad física del adulto mayor constituye la suma de capacidades para realizar por sí mismo actividades indispensables para satisfacer sus necesidades. La dependencia de cualquiera de las actividades básicas e instrumentales de la vida diaria se relaciona con el aumento de la mortalidad en el adulto mayor. El proceso funcional geriátrico inicia cuando un adulto mayor independiente desarrolla limitación en su reserva funcional, la cual se ve manifestada por un estado de vulnerabilidad que da como resultado la discapacidad (Ávila-Fermat et. al. 2012; Secretaría de Salud - México, 2005, citado por (Bejines-Soto et al., 2015)).

Se ha demostrado ampliamente la respuesta positiva a la actividad física por parte de los adultos mayores, debido a que su práctica regular contribuye al mejoramiento de su calidad de vida; sin embargo, la valoración de su condición física establece un paso necesario en el proceso de prescripción del ejercicio físico, así como la evaluación previa al programa de actividad física. Al tener un programa equilibrado éste debe incluir actividades encaminadas a cumplir cada uno de sus objetivos: incrementar la fuerza, aumentar la flexibilidad y elevar la resistencia cardiovascular a partir de tres componentes, el calentamiento, el ejercicio o entrenamiento y la vuelta a la calma, el cual se diseña en función de la adaptación al estado y las necesidades, en términos de salud y problemas médicos, que posee cada adulto mayor (Álvarez \& Alud, 2018).

Está comprobado que la actividad física y el deporte contribuyen a quien los practica un aspecto más saludable y estético; les permite conservar mayor fuerza vital y física, ayudan a mantener y recuperar el equilibrio físico y psíquico, retardan la involución del sistema músculo-esquelético, facilitan la actividad articular y previenen la osteoporosis, evitando las fracturas óseas; interceden en el proceso de envejecimiento cardiovascular, previniendo la arteriosclerosis y mejorando la función endocrina, principalmente la suprarrenal (resistencia y 
adaptación al estrés) y beneficiando el equilibrio neurovegetativo y la actividad psicointelectual (Benavides, García, Fernández, Rodrigues, \& Ariza, 2017).

Dentro de los logros que se destacan en el adulto mayor es que desarrollan un grado de independencia funcional útil, a través de la mejora y el mantenimiento de capacidades físicas mermadas por medio de la aplicación de un programa de actividad física, orientado específicamente a potenciar la fuerza, la flexibilidad, la agilidad y la marcha. (Ávila-Fermat et. al. 2012; Secretaría de Salud - México, 2005, citado por (Bejines-Soto et al., 2015)).

El objetivo de esta investigación fue analizar la relación entre la actividad física y la capacidad funcional en adultos mayores en estudios previamente publicados.

\section{Método}

Diseño

Para cumplir con el objetivo de este trabajo se llevó a cabo una revisión sistemática en las siguientes bases de datos electrónicas de Scientific Electronic Library Online (Scielo), Scopus, Web of Science (WOS) y PEDro, así mismo se consultó el motor de búsqueda Google Académico; utilizando como estrategia recopilar la evidencia científica acerca de los instrumentos más utilizados para analizar la relación entre la actividad física y la capacidad funcional en los adultos mayores, donde se establecieron, como objetivos de búsqueda, los descriptores en lengua inglesa y española. Se estableció como espectro de búsqueda, año 2008 al 2018. Se utilizaron las siguientes palabras claves: functional capacity, physical activity, elderly, ac- tividad física, envejecimiento, capacidad funcional, adulto mayor, programas de actividad física. Se combinaron las palabras clave conjugando el descriptor booleano AND. Se completó la revisión de información utilizando las referencias bibliográficas encontradas en las búsquedas previas, obteniendo así un total de 5200 estudios relacionados con el tema investigado. Los siguientes criterios de inclusión fueron los que se establecieron: estudios que describieran los instrumentos de evaluación para la medición de la condición física funcional y los niveles de actividad física en los adultos mayores, aplicación de programas de actividad física en adultos mayores, que la población fuera mayor de 65 años. Como criterios de exclusión se establecieron: estudios científicos, estudios de caso, estudios con adultos mayores menores de 65 años y publicaciones anteriores a 2008. Estos artículos, se revisaron y se evaluaron de forma exclusiva. Extracción de datos. La información obtenida en cada uno de los estudios estaba dividida en tres tópicos principales:

Datos generales del artículo: Base de datos a la cual pertenece, título, autores, revista, año de publicación, país del estudio y palabras clave.

Población de referencia: Rangos de edad, método de selección de la muestra, tamaño de la muestra, tipo de estudio y criterios de inclusión.

Variables e instrumentos utilizados: "actividad física", "capacidad funcional", adulto mayor, programas de actividad física.

Después del cribado de la información, solo los artículos que cumplían con estos criterios fueron incluidos en la revisión para un total de 53 artículos útiles revisados sobre la temática abordada. 


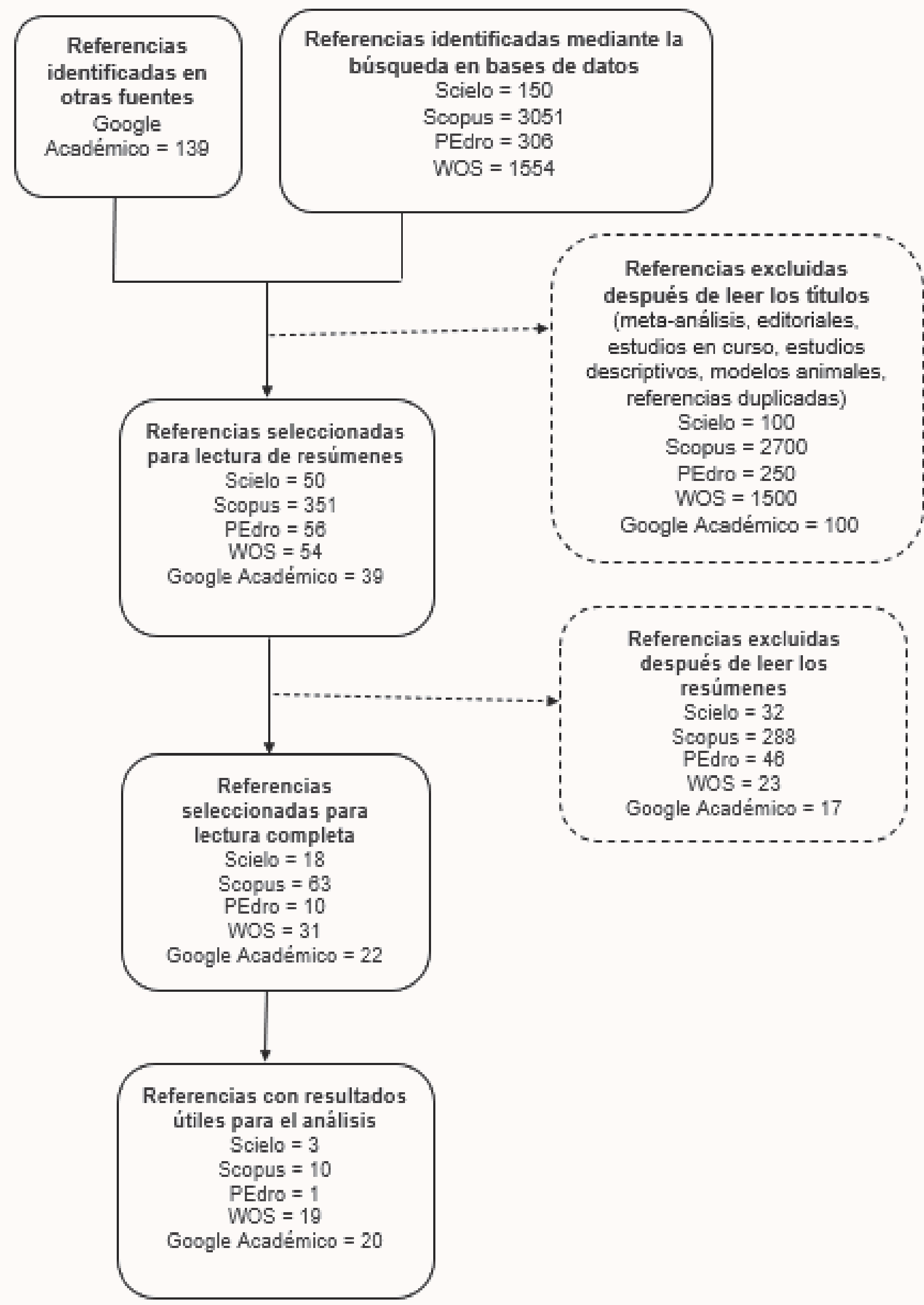

Figura 1. Diagrama de flujo de la búsqueda bibliográfica. Fuente: Elaboración propia. 


\section{Vejez y envejecimiento}

El término "vejez" proviene de la voz latina vetus que deriva de la raíz griega etos que significa "añejo", "años". Por lo general, en su mayoría las personas relacionan la vejez con el tiempo acumulado de vida, como señal del tiempo transcurrido, independientemente de la interpretación que cada grupo o cultura haga de tal signo (Quino-Ávila, Chacón-Serna, \& Vallejo-Castillo, 2017). La vejez se expresa como la desaparición de la juventud; sólo se tiende a estudiarla en términos de pérdidas, sin recalcar los aspectos positivos ni enfocarla como una etapa productiva (Sotomayor \& Torres, 2010).

A partir de los paradigmas de la biología y la medicina, se define la vejez desde dos vertientes mentales: la primera, en función del patrón de referencia cronológica y, la segunda, desde los cambios morfológicos y funcionales de cuya declinación depende el grado de envejecimiento que, además, se manifiesta de manera heterogénea (Moreno, Huerta, \& Albala, 2014). Se ha comprobado que tanto en la ciencia como en la sociedad, han existido diversas dimensiones importantes que se han utilizado para definir la vejez desde tres vertientes, biológica, psicológica y social, que involucra la cuota de participación y de integración social de los viejos (Ramos, Yordi, \& Miranda, 2016).

El proceso de envejecimiento es un fenómeno multifactorial, que aqueja todos los niveles de ordenación biológica, comprendiendo desde las moléculas hasta los sistemas fisiológicos, y en el cual no siempre coincide el fenómeno biológico con el cronológico (Tamez \&
Ribeiro, 2012). Con el transcurso de los años, se reduce la fecundidad y se eleva la tasa de mortalidad, por lo tanto, se comienza a tener menor transcendencia en el conjunto de la sociedad; en compensación, el adulto mayor empiezan a tener mayor representatividad (Cerquera \& Quintero, 2015; Roca, 2016).

Desde otra perspectiva, se define el envejecimiento como un proceso universal, continuo, irreversible, dinámico, progresivo, declinante, heterogéneo $y$, hasta el momento, inevitable, en el que ocurren cambios biopsicosociales resultantes de la interacción de factores genéticos, sociales y culturales, del estilo de vida y de la presencia de enfermedades(Di Domizio, 2015). Dichos cambios definen a los seres humanos cuando ya están mayores; no obstante, se debe ver como un proceso natural, inevitable y no obligatoriamente ligado a estereotipos, como una etapa de la vida apta para "crecer y continuar aprendiendo" (Ramos et al., 2016).

El envejecimiento es un proceso inherente a la vida humana, el cual constituye una experiencia única, heterogénea y su significado varía dependiendo de la connotación que cada cultura haga de él. Se entiende como un fenómeno multidimensional que involucra todas las dimensiones de la vida humana, donde se experimentan cambios de orden físico, psicológico, social, entre otros; por lo cual, se constituye en un espacio ideal para brindar herramientas de intervención que permitan entender el fenómeno de envejecer y asimismo dar alternativas que promuevan la calidad de vida del adulto mayor (Alvarado \& Salazar, 2014). 
Los cambios biológicos únicamente toman sentido en función de una sociedad determinada. La edad es inherente, no a los cambios biológicos exclusivamente, sino también a las percepciones y representaciones que se le atribuyen. Hay una ligera tendencia al declive en las dimensiones del crecimiento personal y el propósito en la vida, según va aumentando la edad, lo cual es consecuencia de un proceso de adaptación, en el que los adultos mayores compensan la pérdida de posibilidades reajustando sus metas.(Aponte, 2015)

El envejecimiento, genera cambios de diferentes índoles, comenzando con alteraciones en diferentes procesos biológicos, psicológicos, cognitivos e inclusive sociales. Otra de las secuelas del envejecimiento es la pérdida de la capacidad funcional, ya que al pasar los años se produce un aumento de los riesgos que predisponen a las personas a perder la autonomía y su funcionalidad. (Rebolledo-Cobos, Silva, Juliao-Castillo, Polo, \& Suarez, 2017).

\section{Capacidad funcional}

La independencia funcional o capacidad funcional es definida como la capacidad de cumplir acciones requeridas en el diario vivir, y de esta forma mantener el cuerpo y subsistir de una manera independiente o autónoma. Cuando la mente y el cuerpo son capaces de llevar a cabo las actividades de la vida cotidiana se dice que la funcionalidad está indemne (Rebolledo-Cobos et al., 2017).

La pérdida de la capacidad funcional del adulto mayor de 65 años de edad se presenta en un $5 \%$ de los casos y en adultos mayores de 80 años se llegan a exhibir en un 50 \%.(Zavala-González \& Domínguez-Sosa, 2011)

Según (Zavala-González, 2011) citando a Encinas, 2000 y Kane, et al. 1991 la funcionalidad se divide en tres categorías:

- Actividades básicas de la vida diaria (ABVD).

- Actividades instrumentales de la vida diaria (AIVD).

- Actividades avanzadas de la vida diaria (AAVD)

Las ABVD hacen referencia principalmente a las actividades que permiten al sujeto ser independiente en su domicilio (alimentación, continencia, transferencia, uso del sanitario, vestido y bañado). Las AIVD permiten una vida independiente en la comunidad (cocinar, realizar las compras, labores domésticas limpieza de la casa, utilización del teléfono, lavado de la ropa, viajar, toma de medicamentos, administración de los gastos personales). Las AAVD permiten desarrollar un papel social (valoran funciones más complejas como la realización de actividades de ocio, religiosas, deportes, trabajos, transporte) (Cerquera, Uribe, Matajira, \& Correa, 2017).

Con el incesante crecimiento de la población de adulto mayor en las sociedades modernas, la exploración de formas de conservar y mejorar las habilidades funcionales de las personas al envejecer, ayudarlas a manejarse independientemente en la comunidad $y$, fundamentalmente, mejorar la calidad de sus vidas, se ha convertido en un asunto cada vez más urgente. 
La incidencia de muchas enfermedades y discapacidades crónicas aumenta con la edad. Sarmiento, 2016).

Normalmente, las personas valoran su estado de salud comparándolo con el de otras personas de su misma edad y condición; así, la valoración de cada persona sobre su propia salud podría describirse como "adaptada a la edad". La esperanza de vida sin discapacidad varía según los distintos países y culturas. La salud de las personas de edad avanzada no debería, y no puede, examinarse simplemente desde el punto de vista de la frecuencia de enfermedades o de la ausencia de las mismas. Incluso si tienen enfermedades, muchas personas mayores se sienten perfectamente sanas porque dichas enfermedades no tienen graves efectos negativos sobre sus vidas diarias (Carpio, Suarez, Almanza, Gómez, \& Quintero, 2012).

Las investigaciones sobre el envejecimiento se han enfocado en la salud, pero últimamente el concepto de capacidad funcional está aclamando cada vez más la atención. Si bien hace mucho que se valora la trascendencia de la funcionalidad sobre la salud y la enfermedad, fue hasta los años 50 que se reconoció su importancia, a medida que se incrementaba la cantidad de personas mayores y discapacitadas, crecía la incidencia de enfermedades crónicas. El valor que debía dársele a la funcionalidad la ratificó la Comisión Estadounidense sobre Enfermedades Crónicas y la OMS, que impulsó el desarrollo de una base científica para medir el estado funcional. Otras investigaciones y programas teóricos examinaron partes claves de la salud funcional: las actividades de la vida diaria (activities of daily living $O A D L$ ), las actividades instrumentales de la vida diaria (instrumental activities of daily living o IADL) y las variables psicológicas y sociales. La capacidad funcional de las personas mayores es crucial con respecto a la forma en que pueden llevar a cabo y soportar las actividades de la vida diaria, que a su vez afecta a su calidad de vida (Medina et al., 2015).

La capacidad funcional, se conceptualiza como la integración de tres campos de la funcionalidad: el biológico, el psicológico (cognitivo y afectivo), y el social. Así, la valoración funcional se deriva de un modelo que observa la forma en que la relación entre estos tres campos contribuye al comportamiento y a la funcionalidad general (Delgado, 2014).

En el adulto mayor, los procesos de desarrollo y envejecimiento pueden dar lugar a grandes diferencias, las mediciones de la salud física pretenden determinar los niveles totales de salud y estado físico. Los indicadores de salud física utilizados habitualmente incluyen diagnósticos y afecciones existentes, los síntomas, las discapacidades, los medicamentos que se consumen, la gravedad de la enfermedad y la cuantificación de los servicios médicos utilizados (por ejemplo, el número de días de hospitalización al año, o la cantidad de días al año en los que no pudo realizar las actividades normales de la vida diaria). La evaluación que realiza la persona de su salud y discapacidad puede incluirse en dichas mediciones. Las escalas de estado funcional estudian las actividades de la vida diaria (lavarse, vestirse, comer, trasladarse, caminar, y la continencia) 
y las actividades instrumentales de la vida diaria (tareas domésticas, hacer la compra, tomar medicinas, utilizar transportes, usar el teléfono, cocinar, y usar y administrar el dinero) que generalmente son necesarias para la vida independiente (Medina et al., 2015).

La competencia funcional también se ha definido como el grado de facilidad con el que una persona piensa, siente, actúa, o se comporta, congruentemente con su entorno y su gasto de energía. A su vez, la salud funcional se ha relacionado con el nivel al que una persona se mantiene a sí misma, la calidad con que desempeña los papeles que le corresponden en la sociedad, el estado intelectual, el estado emocional, la actividad social, y la actitud respecto del mundo y de uno mismo (Rodríguez, Zas, Silva, Sanchoyerto, \& Cervantes, 2014).

Existen grupos de población de un mismo país que están divididos por grandes diferencias en cuanto a morbilidad, mortalidad y capacidad funcional. Un determinante principal de las oportunidades de vida de las personas es su situación económica (Medina et al., 2015).

La capacidad funcional de los sujetos ha sido evaluada clásicamente desde el punto de vista de la actividad física y, centrándonos en esta perspectiva, con base fundamentalmente en elementos fisiológicos, se considera que la máxima función metabólica se logra durante el ejercicio. Por lo cual, es necesario tener un funcionamiento físico adecuado, según los requerimientos de la forma de vida de cada persona lo cual se debe soportar en la independencia (Aires, Giriardi, \& Pinheiro, 2010).
Un adulto mayor sano es aquel capaz de enfrentar el proceso de cambio a un nivel adecuado de adaptabilidad funcional y satisfacción personal, este concepto es clave dentro de la definición de salud; por esto, la OMS propone, como indicador más representativo para este grupo, el estado de independencia funcional. Fillenbaum, ratifican este criterio, proponiendo la evaluación de salud de los adultos mayores en términos de "estatus funcional", asociando el concepto de salud al mantenimiento de la independencia funcional (Durán, Valderrama, Uribe-Rodríguez, \& Uribe, 2008).

Se puede entender por funcionalidad o independencia funcional la capacidad de cumplir acciones requeridas en el diario vivir para subsistir independientemente. El ser humano, como ser social, en todas las etapas de su vida está intercambiando experiencias y conocimientos con su medio, por tanto el aprendizaje no es algo exclusivo de los grupos jóvenes de la población. Los adultos mayores de igual forma pueden continuar en este proceso de aprendizaje como educandos, pero la perspectiva desde la cual se les eduque debe ser diferente, esto es, adecuarse a las características personales por las que está atravesando en esta etapa de su vida (Segovia \& Torres, 2011).

\section{Actividad física}

Existen autores que definen este concepto desde diferentes saberes, pero encaminados a conceptos similares. (Tercedor, Estévez-López, \& Delgado-Fernández, 2012) Precisa a la actividad física como cualquier movimiento producido por los músculos esqueléticos que producen un gasto de energía. 
(Sánchez, 2012), cita a Bouchard et al., 1994 y a Rodríguez, 2001 quiénes definen la actividad física como: "cualquier tipo de ejercicio corporal con un mayor gasto energético que en reposo" y "movimiento corporal producido por los músculos esqueléticos y que produce un gasto energético añadido al metabolismo basal", respectivamente.

Así mismo, el Departamento de Salud y de Servicios Sociales de Estados Unidos(Department of Health and Human Services (HHSS), 2008) publicó que la actividad física se refiere a los movimientos que benefician la salud. Este departamento sugiere algunos criterios necesarios para la correcta práctica de actividad física incentivando a las personas para que puedan llevar a cabo tareas motrices sin llegar a presentar daños o lesiones en su sistema locomotor, sino, por el contrario, puedan obtener beneficios físicos y psicológicos para su vida diaria.

Mientras que el concepto de actividad física que entrega el Colegio Americano de Medicina del Deporte, 2011(ACSM, 2011) es "Cualquier movimiento corporal producido por los músculos que produce un gasto energético por encima de la tasa de metabolismo basal incluyendo actividades de la vida diaria" La Organización Panamericana de la Salud (OPS), considera que la actividad física, está vinculada hacia el campo de la salud y de su calidad de vida, ya que esta se convierte como estrategia que conlleva beneficios en, lo biológico, psicosocial y cognitivo (Vidarte, Vélez, Sandoval, \& Alfonso, 2011).

La (OMS, 2010) define la actividad física como cualquier movimiento corporal producido por los músculos esqueléticos, con el consiguiente consumo de energía. Incluye las actividades realizadas al trabajar, jugar y viajar, las tareas domésticas y las actividades recreativas. El término actividad física no se debería confundir con ejercicio el cual es una subcategoría de actividad física que se planea, está estructurada, es repetitiva y tiene como objetivo mejorar o mantener uno o más componentes del estado físico. La actividad física -tanto moderada como intensa- es beneficiosa para la salud. La OMS considera que la actividad física está ligada a cualquier movimiento corporal producido por el sistema muscular y que a su vez exija a nivel fisiológico un gasto energético.

Aparte del ejercicio, cualquier otra actividad física realizada en el tiempo de ocio, para desplazarse de un lugar a otro o como parte del trabajo, es igualmente beneficiosa para la salud.

La actividad física recomendada varía según la clasificación de grupos etarios que maneja la OMS, entre los cuales destacamos los siguientes:

Para adultos de 18 a 64 años de edad: Practicar al menos 150 minutos semanales de actividad física moderada, o al menos 75 minutos semanales de actividad física intensa, o una combinación equivalente entre actividad moderada e intensa. Para obtener mayores beneficios para la salud los adultos deben llegar a 300 minutos semanales de actividad física modera$\mathrm{da}$, o su equivalente. Conviene realizar las actividades de fortalecimiento muscular 2 o más días a la semana y de tal manera que se ejerciten grandes conjuntos musculares. 
Para adultos de 65 o más años de edad: Practicar al menos 150 minutos semanales de actividad física moderada, o al menos 75 minutos semanales de actividad física intensa, o una combinación equivalente entre actividad moderada e intensa. Para obtener mayores beneficios para la salud estas personas deben llegar a 300 minutos semanales de actividad física modera$\mathrm{da}$, o su equivalente. Las personas con problemas de movilidad deben practicar actividad física para mejorar su equilibrio y prevenir caídas por lo menos 3 días a la semana. Conviene realizar las actividades de fortalecimiento muscular 2 o más días a la semana y de tal manera que se ejerciten grandes conjuntos musculares.

La intensidad con que se practican distintas formas de actividad física varía según las personas. Para que beneficie a la salud cardiorrespiratoria, toda actividad debe realizarse en periodos de al menos 10 minutos de duración.

Incorporar la actividad física como una herramienta que otorga múltiples beneficios en la salud, como, mejorar la fuerza muscular, lo que genera efectos positivos sobre la capacidad funcional de la persona, principalmente en las funciones básicas de la vida diaria; mejora de flexibilidad y movilidad articular, que ayudaría a un mejor desplazamiento, equilibrio, disminuyendo los riesgos de caídas entre otros, los cuales, a largo plazo conllevan a una mayor independencia y mejor calidad de vida.

Beneficios de la práctica de actividad física.

La práctica de actividad física regular de intensidad moderada -como caminar, montar en bicicleta o hacer deporte- posee considerables beneficios para la salud. En las diferentes edades, los beneficios de la actividad física contrarrestan posibles daños provocados, como ejemplo, por accidentes. Llevar a cabo algún tipo de actividad física es mejor que no realizar ninguna. Convirtiéndonos más activos a lo largo del día de formas relativamente simples podemos alcanzar fácilmente los niveles recomendados de actividad física (OMS, 2010).

Heredia, 2006 citado por (Jiménez, Núñez \& Coto, 2018), quien menciona acciones "encaminadas a conseguir cada uno de los tres objetivos de una buena actividad física: aumentar la flexibilidad, incrementar la fuerza y elevar la resistencia cardiovascular sobre los tres componentes de cada sesión de ejercicios: el calentamiento, el ejercicio o entrenamiento y el enfriamiento". Cuando se prescribe ejercicio para estas personas, es de vital importancia trabajar todos los aspectos, para conseguir equilibrio, sin sobrecargar alguna o descuidar otra.

(Jiménez, Núñez \& Coto, 2018) considera que: "El ejercicio físico practicado regularmente en la tercera edad y en niveles adecuados contribuye a:

- Mejorar la capacidad para el auto cuidado

- Favorecer la integración del esquema corporal

- Propiciar bienestar general

- Conservar más ágiles y atentos los sentidos

- Facilitar las relaciones inter-generacionales 
- Aumentar los contactos y su participación social.

- Inducir cambios positivos en el estilo de vida de los adultos mayores

- Incrementar la calidad del sueño

- Disminuir la ansiedad, el insomnio y la depresión

- Reforzar la actividad intelectual, gracias a la buena oxigenación cerebral

- Contribuir en gran manera al equilibrio psico-afectivo

- Prevenir caídas

- Incrementar la capacidad aeróbica, la fuerza muscular y la flexibilidad

- Disminuir el riesgo de enfermedad cardiovascular

- Hacer más efectiva la contracción cardíaca

- Frenar la atrofia muscular

- Favorecer la movilidad articular

- Evitar la descalcificación ósea

- Aumentar la eliminación de colesterol, disminuyendo el riesgo de

- arterio-esclerosis e hipertensión

- Reducir el riesgo de formación de coágulos en los vasos y por tanto de trombosis y embolias.

- Aumentar la capacidad respiratoria y la oxigenación de la sangre

- Evitar la obesidad

- Mejorar la capacidad funcional de aquellos individuos que presentan un déficit en la realización de las AVD.

- Incrementar la longevidad.

\section{Actividad física y capacidad funcional}

En cuanto a la relación de la actividad física y la capacidad funcional, hay diversos estudios en los que se demostraron la efectividad del ejercicio físico en el aumento de la capacidad funcional al momento de mantener el equilibrio, tanto dinámico como estático. Se obtuvieron diferencias estadísticamente significativas en el mantenimiento del equilibrio en determinadas posiciones (Fonseca et al., 2014; Leiróz-Rodríguez, Romo-Pérez, Arce, \& García-Soidán, 2017).

Incluir la valoración funcional del adulto mayor sirve para orientar los planes o estrategias de intervención en actividad física, describen el perfil morfológico y funcional, y analizan la relación de la velocidad de movimiento y el equilibrio dinámico con las características antropométricas(Kruleske, Salmaso, \& Da Silva, 2013).

En un trabajo realizado por (Silva-Zemanate, Pérez-Solarte, Fernández-Cerón, \& Tovar-Ruíz, 2014) se utilizó el arte marcial del Tai Chi para determinar los efectos en la capacidad funcional de los adultos mayores, observaron que dicha capacidad mejoró en todos los aspectos evaluados, con diferencias significativas en la velocidad de marcha y el equilibrio estático y el dinámico; la capacidad cognoscitiva mejoró en todos los ítems evaluados, con diferencias estadísticamente significativas en el de la memoria, la atención-cálculo y el lenguaje; todos los participantes alcanzaron una completa funcionalidad en las actividades básicas cotidianas.

El envejecimiento activo está encaminado a mejorar las oportunidades de bienestar del adulto mayor en los aspectos psicológicos, físicos y sociales, con el objetivo de ampliar la esperanza de vida saludable; para alcanzar este propósito, la actividad física y sus beneficios son la herramienta ideal, mejorando su funcionalidad, la cual se define como la aptitud para ejecutar 
eficientemente las actividades básicas e instrumentales y de esta forma, ser independiente; las restricciones en su ejecución son un indicador de alarma para la salud, por lo que se afecta directamente la calidad de vida del adulto mayor (Quino-Ávila et al., 2017).

Un mayor nivel de actividad física está relacionado con la participación en las actividades físicas moderadas, que pueden retrasar el declive funcional y reducir el riesgo de padecer enfermedades, por tanto, mantener un estilo de vida activo garantizará una mayor independencia funcional (Landinez et al. 2012).

Programa de ejercicio físico multicomponente en el adulto mayor.

ACSM, 2014 menciona que los diversos programas de ejercicio físico realizados en la comunidad para los adultos mayores han adquirido popularidad. El Colegio Americano de Medicina del Deporte (ACSM, 2011) sugiere que las personas que deseen iniciar un programa vigoroso de ejercicios deben ser supervisados por médicos, la evaluación para todos puede ser impráctica y constituir un obstáculo para su realización. Si la recomendación es caminar o participar en programas de baja intensidad, las pruebas probablemente no sean necesarias. Los componentes del programa de ejercicio físico deberán incluir periodos de: Calentamiento. Ejercicio aeróbico. Entrenamiento de la fuerza. Periodo de enfriamiento.

Las etapas de calentamiento y enfriamiento son importantes en los adultos mayores porque la adaptación de la frecuencia cardiaca, tensión arterial y frecuencia respiratoria es más lenta. El ejercicio aeróbico (EA) incluirá preferentemente actividades de bajo impacto (caminar, pedalear, nadar, remar, subir escaleras). En el caso del entrenamiento de fuerza (EF), éste debe estar encaminado a los grandes planos musculares que componen los hombros, brazos, columna, cadera y piernas, los cuales son importantes para las actividades de la vida diaria (Ceballos, 2012).

Los programas que abarcan ejercicios de flexibilidad, resistencia, equilibrio y fuerza, forman las intervenciones más efectivas en la mejoría de la condición física y el estado de salud global de los adultos mayores. Estas intervenciones disminuyen la morbi-mortalidad, la incidencia y el riesgo de caídas; previniendo el deterioro funcional y la discapacidad. Las mejorías de la capacidad funcional son innegables cuando la intervención está dirigida a más de un componente de la condición física (resistencia, fuerza y equilibrio) comparado con un único tipo de ejercicio físico. Por lo tanto, los diferentes estímulos aplicados en un mismo programa pueden desencadenar mayores ganancias funcionales que aplicados individualmente (Chin, Uffelen, Riphagen, \& Mechelen, 2008; Daniels, Van Rossum, De Witte, Kempen, $\&$ Van Den Heuvel, 2008).

En un estudio realizado por (Cadore et al., 2014) se observó que después de realizar un entrenamiento definido durante un período de 12 semanas, adultos mayores de 90 años mejoraron su fuerza, potencia y masa muscular, lo que se manifestó en mayor habilidad para levantarse de la silla, un aumento de la velocidad al caminar, reducción significativa de la incidencia de caídas, 
mejora del equilibrio y mejora significativa de la potencia y masa muscular en las extremidades inferiores. Este programa de entrenamiento mejoró la capacidad funcional, la reducción del riesgo de caídas y potencia muscular. Desde un punto de vista práctico, los resultados obtenidos en el estudio recalcan la importancia de implementar programas de actividad física en adultos mayores, ejercicios para desarrollar la fuerza muscular, el equilibrio y la marcha.

Lo anterior refleja el beneficio de este tipo de programas en adultos mayores, sin embargo la progresión del entrenamiento y la inclusión de diferentes estímulos debería seguir los principios básicos del entrenamiento y realizarse de manera lenta y progresiva (Izquierdo, Cadore, \& Casas, 2014).

\section{Calentamiento y enfriamento}

Iniciación. 5 minutos de calentamiento son suficientes; pueden incluirse actividades como estiramiento, caminar despacio e incluso pedalear. El calentamiento aumentará el gasto cardiaco y mejora la perfusión central y periférica.

Como fase de enfriamiento puede realizarse una caminata lenta 0 , incluso, más estiramiento. Esta fase debe durar de 10 a 15 minutos e idealmente bajo observación. Esta parte del programa permitirá el retorno venoso a pesar de la vasodilatación periférica, además de disminuir la incidencia de complicaciones postejercicio asociadas al aumento de catecolaminas, hipotensión y pérdida de calor. Vale la pena mencionar que tener una técnica adecuada de respiración durante el ejercicio, disminuirá el estrés cardiovascular, especialmente en el EF; en este caso, se deberá inhalar antes de levantar el peso, exhalar al levantarlo y al bajar, nuevamente, se debe inhalar. (E Cadore \& M Izquierdo, 2013; Evans, 1997; Vidarte, Quintero, \& Herazo, 2012; Vidarte et al., 2011).

De acuerdo al (ACSM, 2014), la intensidad y duración del entrenamiento debe tener las siguientes características: habitualmente, el programa de ejercicio avanzará en tres etapas: Programa de inicio, lenta progresión y programa de mantenimiento.

A continuación se numeran las sugerencias del Colegio Americano de Medicina del Deporte (ACSM, 2011):

1. La frecuencia del entrenamiento debe ser, al menos, tres veces por semana.

2. La intensidad del ejercicio aeróbico (EA) recomendada es de 50 a $85 \%$ del VO2max o de la FC max.

3. La duración del EA debe ser de 20 a 60 minutos, dependiendo de la intensidad de la actividad.

4. El EA debe ser cualquier actividad que use los grandes grupos musculares de forma rítmica y continua como lo es caminar, trotar, correr, bicicleta, nadar, subir escaleras y cierto tipo de danza.

5. El entrenamiento de fuerza (EF) de moderada intensidad es suficiente para mantener y desarrollar la masa muscular, así como la densidad ósea, y debe de incluirse en el programa.

6. Se recomienda una serie de ocho a 12 repeticiones de ocho a 10 ejercicios que trabajen los grupos musculares principales, al menos dos veces por semana. 
7. La intensidad progresará de forma gradual, habitualmente cada dos a tres semanas.

8. El programa total de ejercicio idealmente no durará más de una hora, por lo que se usarán sesiones alternadas para combinar los ejercicios.

Existen ciertas recomendaciones especiales para iniciar un programa de fuerza, las cuales incluyen: iniciar con el peso más ligero del aparato 0 , si se usa 1RM, empezar de 30 a $40 \%$; si levanta el peso cómodamente más de 15 veces o el paciente lo nota ligero, se aumentará $5 \%$ la próxima serie; la meta es que haga entre 8 y 12 repeticiones. Con estas recomendaciones, los cambios en la capacidad aeróbica y la fuerza del adulto mayor son apreciables en etapas muy tempranas del programa de ejercicios físicos (Benavides et al., 2017; E Cadore \& M Izquierdo, 2013).

\section{Discusión}

En un análisis de todos los artículos revisados se evidenció que la actividad física en los adultos mayores (OMS, 2010; Tercedor et al., 2012) debe ser considerada como una de las principales estrategias de promoción de la salud y prevención de la enfermedad, la cual se debería implementar desde temprana edad para evitar la aparición de enfermedades crónicas en la edad adulta, lo que a su vez conlleva a prevenir la dependencia funcional (Rebolledo-Cobos et al., 2017) (Cadore et al., 2014; Zavala-González \& Domínguez-Sosa, 2011) debido a que la actividad física ayuda a prevenir la fragilidad y a mejorar el equilibrio, la fuerza y la marcha en los adultos mayores, lo cual conlleva a que el adulto mayor sienta mayor seguridad al realizar las diferentes actividades de la vida diaria.

En el consenso en la literatura se obtienen resultados favorables acerca de los beneficios y seguridad de las intervenciones con actividad física en adultos mayores. Los efectos encontrados permiten recomendar la prescripción de intervenciones que incluyan la práctica de al menos 150 min por semana de actividades de intensidad moderada con el ánimo de lograr mejoras en desenlaces como la calidad de vida relacionada con la salud (CVRS) y las cifras tensionales, las cuales se muestran como marcadores importantes de progreso clínico de los pacientes, así como de la carga asociada a la enfermedad y la mortalidad (Cobo-Mejía, Prieto-Peralta, \& Sandoval-Cuellar, 2016).

Dentro de las principales estrategias para lograr un envejecimiento activo se encuentra la actividad física, optimizando el bienestar del individuo en todas las esferas; además de intervenir en la capacidad funcional beneficiando o limitando la realización de actividades de la vida diaria básica y cotidiana en forma funcional, autónoma e independiente (Quino \& Chacón, 2018).

Los resultados permiten aseverar que la edad es determinante en la capacidad del adulto mayor para realizar actividades básicas de la vida diarias, es decir, que la funcionalidad disminuye con la edad, ya que en esta etapa, conforme pasan los años se van produciendo cambios en el organismo, tales como: alteraciones en los diversos órganos, en los sentidos, en los patrones 
del sueño, el sistema inmunitario, entre otros; lo que reduce la capacidad de la persona para ejecutar las actividades del diario vivir, disminuyendo considerablemente su nivel de autonomía. Por otro lado, se puede afirmar que el sexo no es determinante en la capacidad funcional del adulto mayor. Por tanto, la capacidad funcional es indistinta del sexo; dependerá del estilo de vida que haya llevado el adulto mayor a los largo de su vida y de la edad en la que se encuentre, lo que le permitirá realizar sus actividades con total independencia (Delgado, 2014).

\section{Conclusión}

La búsqueda permitió exponer las diferentes formas de abordaje del tema, desde nuevas perspectivas de evaluación y tratamiento y se proponen cambios en las estrategias de intervención y evaluación, para poder modificar y restaurar los deterioros producidos en el proceso de envejecimiento.

La actividad física es el único método probado y seguro que puede mantener la condición física de un individuo, además de impedir e incluso revertir los cambios en la composición corporal asociados al envejecimiento, particularmente la pérdida de músculo esquelético. Los adultos mayores son el grupo de la población que más se beneficia de esta actividad y la principal diferencia con los programas de actividad física realizada por jóvenes es la forma como se aplica, es decir, iniciar bajo y avanzar lento.

El entrenamiento de la coordinación, de la capacidad funcional, del equilibrio, de la fuerza muscular debe ser una meta, pero sostenible. Se recomienda trabajar en grupos o en familia ya que mejorará el apego y la permanencia en los programas. La práctica continua de actividad física mejorará la funcionalidad e independencia, así como también será benéfico para el estado de ánimo y la calidad de vida del adulto mayor.

La actividad física y la capacidad funcional en el adulto mayor poseen notables beneficios, relacionados con factores fisiológicos, psicológicos y sociales. Sin embargo, estos beneficios dependerán estrictamente de cada individuo $y$, por supuesto, del trabajo planeado y su objetivo, adaptando la planificación a las particularidades del sujeto.

Se debe recomendar un programa de rehabilitación realista, esto ayudará a que los adultos mayores conserven y/o recuperen la funcionalidad.

Difundir el material resultado del presente estudio, en los distintos centros de salud y universitarios donde exista atención al adulto mayor para promover un mejor manejo de la actividad física y la capacidad funcional de estas personas. Se incita a futuros investigadores a llevar a cabo estudios en poblaciones de edad avanzada para seguir obteniendo registros y resultados en nuestro país de los beneficios de la actividad física en la salud del adulto mayor. 


\section{Referencias}

ACSM, A. C. o. S. M. (2011). Quantity and Quality of Exercise for Developing and Maintaining Cardiorespiratory, Musculoskeletal, and Neuromotor Fitness in Apparently Healthy Adults: Guidance for Prescribing Exercise. ACSM. doi: 10.1249/MSS. 0b013e318213fefb

ACSM, A. C. o. S. M. (2014). Manual ACSM para la valoración y prescripción del ejercicio España: Paidotribo.

Aires, M., Giriardi, L., \& Pinheiro, E. (2010). Capacidad funcional de ancianos con edad avanzada: estudio comparativo en tres regiones de Río Grande del Sur. Revista Latino-Americana de Enfermagem, 18 (1), 1-8.

Alvarado, A., \& Salazar, A. (2014). Análisis del Concepto de Envejecimiento. Gerokomos, 25(2), 5762. doi: dx.doi.org/10.4321/S1134928X2014000200002

Álvarez, E., \& Alud, A. (2018). La actividad física y sus beneficios físicos como estrategia de inclusión social del adulto mayor. Revista Inclusión \& Desarrollo, 5 (1), 23-36.

Aponte, V. (2015). Calidad de vida en la tercera edad. Ajayu. Órgano de Difusión Científica del Departamento de Psicología de la Universidad Católica Boliviana "San Pablo", 13 (2), 152-182.

Araya, A. (2012). Calidad de Vida en la Vejez (S. I. S.A. Ed. Primera ed.). Santiago de Chile: Pontificia Universidad Católica de Chile.
Bejines-Soto, M., Velasco-Rodríguez, R., García-Ortiz, L., Barajas-Martínez, A., Aguilar-Núñez, L., \& Rodríguez, M. (2015). Valoración de la capacidad funcional del adulto mayor residente en casa hogar. Revista Enfermería Instituto Mexicano Seguro Social, 23 (1), 9-15.

Benavides, C., García, J., Fernández, J., Rodrigues, D., \& Ariza, J. (2017). Condición física, nivel de actividad física y capacidad funcional en el adulto mayor: Instrumentos para su cuantificación. Revista U.D.C.A Actualidad \& Divulgación Científica, 20 (2), 255-265.

Cadore, E., Casas-Herrero, A., Zambom-Ferraresi, F., Idoate, F., Millor, N., Gómez, M., . . Izquierdo, M. (2014). Multicomponent exercises including muscle power training enhance muscle mass, power output, and functional outcomes in institutionalized frail nonagenarians. AGE, 36, 773-785. doi: 10.1007/s11357-013-9586-z

Cadore, E., \& Izquierdo, M. (2013). How to simultaneously optimize muscle strength, power, functional capacity, and cardiovascular gains in the elderly: an update. AGE, 35 (6), 2329-2344. doi: 10.1007/s11357012-9503-x

Cadore, E., \& Izquierdo, M. (2013). New Strategies for the Concurrent Strength-, Power-, and Endurance- Training Prescription in Elderly Individuals. JAMDA, 14(8), 623-624. doi: dx.doi.org/10.1016/j. jamda.2013.04.008 
Carpio, S., Suarez, A., Almanza, M., Gómez, L., \& Quintero, M. (2012). Caracterización de las actividades básicas de la vida diaria ABVD en un grupo de adultos mayores institucionalizados Revista Salud en Movimiento, 4 (1), 4-18.

Ceballos, O. (2012). Actividad física en el adulto mayor. México: Manual Moderno.

Cerquera, A., \& Quintero, M. (2015). Reflexiones grupales en Gerontología: El Envejecimiento normal y patológico. Revista Virtual Universidad Católica del Norte, 45, 173-180.

Cerquera, A., Uribe, A., Matajira, Y., \& Correa, H. (2017). Dependencia funcional y dolor crónico asociados a la calidad de vida del adulto mayor. Psicogente, 20 (38), 398-409.

Cobo-Mejía, E., Prieto-Peralta, M., \& Sandoval-Cuellar, C. (2016). Efectos de la actividad física en la calidad de vida relacionada con la salud en adultos con hipertensión arterial sistémica: revisión sistemática y metaanálisis. Rehabilitación, 50 (3), 139-149. doi: dx.doi.org/10.1016/j.rh.2015.12.004

Chin, M., Uffelen, J., Riphagen, I., \& Mechelen, W. (2008). The Functional Effects of Physical Exercise Training in Frail Older People. Sportes Medicine, 38 (9), 781-793.

Daniels, R., Van Rossum, E., De Witte, L., Kempen, G., \& Van Den Heuvel, W. (2008). Interventions to prevent disability in frail community-dwelling elderly: a systematic review. BioMed Central Ltda, 8, 278. doi: doi:10.1186/1472-6963-8-278

Delgado, T. (2014). Capacidad funcional del adulto mayor y su relación con sus características sociodemográficas, Centro de Atención Geronto Geriátrico "Ignacia ROdulfo vda de Canevaro", Lima 2014 Revista Peruana de Obstetricia en Enfermería, 10 (1).

Department of Health and Human Services (HHSS). (2008). Physical Activity Guidelines for Americans. Washington DC: Retrieved from https:// health.gov/paguidelines/pdf/paguide.pdf.

Di Domizio, D. (2015). Educación física con adultos mayores: algunas refleciones desde la gerontología crítica. Revista Umbral, 10, 64-75.

Durán, D., Valderrama, L., Uribe-Rodríguez, A., \& Uribe, J. (2008). Integración social y habilidades funcionales en adultos mayores. Universitas Psychologica, 7 (1), 263-270.

Evans, W. (1997). Functional and Metabolic Consequences of Sarcopenia. The Journal of Nutrition, 127(5), 998S-1003S. doi: doi.org/10.1093/ jn/127.5.998S

Fonseca, A., Ariza, C., Boneth, M., Parra, J., Rojas, R., \& ANgarita, Y. (2014). Reproducibilidad de una prueba para la evaluación funcional del balance dinámico y la agilidad deladulto mayor. Iatreia, 27 (3), 290-298.

Izquierdo, M., Cadore, E., \& Casas, A. (2014). Ejercicio físico en el anciano frágil: una manera eficaz de prevenir la dependencia. Kronos, 13 (1), 2-14. 
Jiménez, Y., Núñez, M. \& Coto, E. (2018). La actividad física para el adulto mayor en el medio natural. InterSedes: Revista de las Sedes Regionales. XIV (27), 168-181.

Kruleske, C., Salmaso, C., \& Da Silva, R. (2013). Fear of falling and self-perception of health in older participants and non-participants of physical activity programs. Motriz Revista de Educação Física, 19 (4), 763-769.

Landinez, N., Contreras, K., Castro, A. (2012). Proceso de envejecimiento, ejercicio y fisioterapia. Rev. Cubana de Salud Pública. 38(4):562-580.

Leiróz-Rodríguez, R., Romo-Pérez, V., Arce, M., \& García-Soidán, J. (2017). Relación entre composición corporal y movimientos producidos durante la marcha en personas mayores. Fisioterapia, 39 (3), 101-107. doi: dx. doi.org/10.1016/j.ft.2016.11.002

Medina, J., Gámez, C., Rivera, M., Bazaldúa, L., Baca, J., Barrón, U., . . . Fuentes, S. (2015). Intervenciones de Enfermería para el Cuidado del Adulto Mayor con Deterioro Cognoscitivo. México: Instituto Mexicano del Seguro Social.

Moreno, X., Huerta, M., \& Albala, C. (2014). Autopercepción de salud general y mortalidad en adultos mayores. Gaceta Sanitaria, 28 (3)(246-252). doi: http://dx.doi.org/10.1016/j.gaceta.2013.07.006

OMS, O. M. d. I. S. (2010). Recomendaciones mundiales sobre actividad física y salud. Suiza.
Organización Mundial de la Salud (OMS). (2015). Informe Mundial sobre el Envejecimiento y la Salud. USA: OMS.

Quino-Ávila, A., Chacón-Serna, M., \& Vallejo-Castillo, L. (2017). Capacidad funcional del anciano relacionada con la actividad física. Revista de Investigación en Salud, 4 (1), 86-103. doi: dx.doi.org/10.24267/23897325.199

Quino, A., \& Chacón, M. (2018). Capacidad funcional relacionada con actividad física del adulto mayor en Tunja, Colombia. Horizonte Sanitario, 17 (1), 59-68. doi: 10.19136/ hs.a17n1.1870

Ramos, A., Yordi, M., \& Miranda, M. (2016). El envejecimiento activo: importancia de su promoción para sociedades envejecidas Revista Electrónica Archivo Médico de Camagüey, 20 (3), 330-337.

Rebolledo-Cobos, R., Silva, C., Juliao-Castillo, J., Polo, R., \& Suarez, O. (2017). Functional implications of strength training on older adults: a literature review. Archivos de Medicina del Deporte, 34 (1), 31-39.

Roca, R. (2016). Actividad física y salud en el adulto mayor de seis países latinoamericanos Review. 17, 77-86.

Rodríguez, J., Zas, V., Silva, E., Sanchoyerto, R., \& Cervantes, M. (2014). Evaluación geriátrica integral, importancia, ventajas y beneficios en el manejo del adulto mayor Panorama Cuba y Salud, 9 (1), 35-41. 
Sánchez, A. (2012). Acondicionamiento físico, calidad de vida y condición física. Un estudio longitudinal en mujeres mayores sedentarias. (Doctorado), Universidad de Extremadura, España. Retrieved from https:// dialnet.unirioja.es/servlet/tesis?codigo $=25770$

Sarmiento, L. (2016). Envejecimiento y actividad físico deportiva (AFD). International Journal of Developmental and Educational Psychology, 1 (2), 135-142.

Segovia, M., \& Torres, E. (2011). Funcionalidad del adulto mayor y cuidado enfermero. Gerokomos, 22 (4), 162-166.

Silva-Zemanate, M., Pérez-Solarte, S., Fernández-Cerón, M., \& Tovar-Ruíz, L. (2014). Efectos del Tai Chi en la capacidad funcional de un grupo de mujeres ancianas. Revista Ciencias de Salud, 12 (3), 353-369. doi: dx.doi. org/10.12804/revsalud12.03.2014.05

Sotomayor, U., \& Torres, J. (2010). Migración y vejez: Una mirada desde las representaciones sociales. CIMEXUS, 5 (2), 149-161.

Tamez, B., \& Ribeiro, M. (2012). El proceso de envejecimiento y su impacto socio-familiar. Revista Latinoamericana de Estudios de Familia, 4, 11-30.

Tercedor, P., Estévez-López, F., \& Delgado-Fernández, M. (2012). Recomendaciones de actividad física para adultos sanos. Revisión y situación actual. Journal of Sport and Health Research, 4 (3), 233-244.
Vidarte, J., Quintero, M., \& Herazo, Y. (2012). Efectos del ejercicio físico en la condición físico funcional y la estabilidad en adultos mayores. Hacia la Promoción de la Salud, 17 (2), 79-90.

Vidarte, J., Vélez, C., Sandoval, C., \& Alfonso, M. (2011). Actividad física: Estrategia de promoción de la salud. Hacia la Promoción de la Salud, 16 (1), 202-218.

Zavala-González, M., \& Domínguez-Sosa, G. (2011). Funcionalidad para la vida diaria en adultos mayores. 